\title{
Potential, Erfolge und Herausforderungen der Agenten-basierten Modellierung in den Wirtschaftswissenschaften: Kommentar zum Beitrag von Herbert Dawid
}

\author{
Gisela Kubon-Gilke
}

Online publiziert: 22. Februar 2019

(C) List-Gesellschaft e.V. 2019

\section{Einleitung}

Ziel des Beitrags von Herbert Dawid ist es, die Anwendung Agenten-basierter Modelle (ABM) für die Wirtschaftswissenschaften darzustellen, deren Bedeutung herauszuarbeiten, aber auch Herausforderungen zu benennen. Der Aufsatz ist in der Kürze und Klarheit der Argumente überaus informativ und hilfreich, um die Modellierungsmethode einschließlich ihrer verschiedenen Interpretationen und Fokussierungen verstehen und einschätzen zu können. Die Beschreibungen, Systematisierungen und Analysen Dawids sollen deshalb nachfolgend auch nur am Rande und im Rahmen weitergehender Fragen angesprochen werden, da in dem Aufsatz die vorhandene und relevante Literatur in einem exzellenten Überblick und in sinnvoller Strukturierung vorgestellt wird, Anwendungsfelder identifiziert werden und der gesamte Ansatz systematisch beurteilt wird. Statt immanenter Kritik werden in diesem Kommentar deshalb zwei eher ergänzende und vorgelagerte Grundsatzfragen angeschnitten, erstens zur Bedeutung methodologischer Überlegungen für die Anwendung der ABM und zweitens damit zusammenhängend zu psychologischen Grundfragen und zur konkreten Basis bei der Auswahl von Annahmen über individuelles Verhalten und die Interaktionen von Individuen.

This commentary article refers to the article available online at https://doi.org/10.1007/s41025-01900131-w. 


\section{Methodologische Anmerkungen}

Agenten-basierte Modelle werden u. a. deshalb als sinnvolle Methode angesehen, weil die ansonsten in der vermeintlichen Mainstream-Ökonomik verwendeten Annahmen über menschliches Verhalten als unzureichend bis falsch beschrieben werden. Dawid charakterisiert es so, dass individuelles Entscheiden und Handeln tatsächlich oft nicht mit rationalen Erwartungen und optimalem Verhalten in Einklang gebracht werden können und den komplexen Interaktionen (heterogener) Akteure in traditionellen Modellen nicht viel Aufmerksamkeit geschenkt würde. Das ist zweifellos korrekt, dennoch sollte beachtet werden, dass Argumente pro Homo oeconomicus in diesem Verständnis gar nicht unbedingt darauf fußen, dass den Menschen tatsächlich solches Verhalten unterstellt würde. Vielmehr ist es im Kern eine Aussage im als-ob-Sinne mit dem Argument, dass Wettbewerbskräfte so stark kanalisieren, so dass die Ergebnisse der Konkurrenz weitgehend unabhängig von individuellen Verhaltensdispositionen sind und man die einfachste Annahme (Optimierung) nehmen könne, um diese Wettbewerbsergebnisse geeignet zu rekonstruieren und auch, um Prognosen über zukünftige Entwicklungen abgeben zu können (z. B. Alchian 1950). Auf analytisch komplexer hergeleitete Begründungen der Optimierungsthesen im Vergleich zur Unterstellung naiver psychologischer Dispositionen hat bereits John Stuart Mill hingewiesen (vgl. zusammenfassend Kubon-Gilke et al. 2018, S. $268 \mathrm{f}$. [Nutzinger]). Wenn die Optimierungshypothese aber aus gänzlich anderen Überlegungen und nicht auf der Grundlage naiver Psychologie gerechtfertigt wird, dann erfordert es hintergründigere Kritikbegründungen als die etwas lapidare Feststellung, dass die Menschen weder tatsächlich als Homo oeconomicus agieren noch dass Wechselwirkungen individueller Entscheidungen und Regeln von Bedeutung sind.

Die Überlegungen zur Sinnhaftigkeit der Optimierungsannahme kann man in jeweils konkreten Fällen ökonomischer Analysen kritisieren, wenn man je nach Analysegegenstand begründet bezweifeln kann, dass Wettbewerbsergebnisse tatsächlich unabhängig von Verhaltensdispositionen der Menschen und ihren Interaktionen sind. Das aber hängt wiederum von der konkreten ökonomischen Frage, dem betrachteten Zeithorizont und der darauf fußenden sinnvollen Auswahl exogener und endogener Modellgrößen ab. Per se kann man die als-ob-Annahme streng optimierenden Verhaltens weder komplett verwerfen noch grundsätzlich rechtfertigen, d.h. in bestimmten Wettbewerbsumgebungen kann die Prognoseleistung via Homo oeconomicus recht gut sein (kurzfristige Wirkung von Mietpreisbremsen etwa). In anderen Zusammenhängen ist die Prognose schlecht und z.T. irreführend. Das kann beispielsweise der Fall sein, wenn in Arbeitsmarktzusammenhängen übersehen wird, dass sich Einstellungen, Motive und Verhalten systematisch mit modellendogenen Variablen verändern und dementsprechend deshalb auch endogen und nicht exogen behandelt werden sollten. In letzterem Fall benötigt man komplexere Analyseinstrumente. Das gilt auch etwa für Betrachtungen von Marktdynamiken, bei denen Innovationen u. a. eine Rolle spielen.

Die Diskussion über als-ob-Rechtfertigungen von Optimierungsannahmen führt zu sehr grundsätzlichen methodologischen Problemen der Ökonomik, was man sich gut an der Konstruktion des repräsentativen Agenten veranschaulichen kann. Man 
sollte dabei die konkreten Modelle zu rationalen Erwartungen sowie ihren Begründungen und Interpretationen keinesfalls mit der Grundsatzidee repräsentativer Agenten vermischen. Die Modelle rationaler Erwartungen können aus guten Gründen systematisch kritisiert werden, das liegt aber an einem sehr speziellen und verkürzten Verständnis der Konstruktion repräsentativer Agenten in diesen Modellen. Das von Dawid angeführte Kirmansche Argument (Kirman 1992) ist sicherlich zutreffend, dass sich im Gruppenaggregat individuelle Verhaltensregeln nicht zwangsläufig widerspiegeln müssen. Der Rückgriff auf individuelles Verhalten entspricht allerdings auch keineswegs der Grundsatzidee bei der Konstruktion des repräsentativen Agenten, sondern nur speziellen Interpretationen und Anwendungen. Unzulässigerweise werden dabei nämlich repräsentative und typische Agenten gleichgesetzt. Typische Agenten sind nur dann repräsentative, wenn gegenseitige Abhängigkeiten keine große Rolle spielen oder sich gegenseitig neutralisieren. In anderen Fällen ist die Konstruktion eines repräsentativen Agenten komplexer und geht unmittelbar von der Aggregateinheit aus, indem gefragt wird, ob das Aggregat bestimmten Regeln folgt und wie man diese analytisch geeignet erfassen kann. Das kann, muss aber nicht optimierendes Verhalten eines typischen Akteurs sein, da es auf die Interaktionen ankommt, die das Aggregat bestimmen und über das man bestimmte Annahmen trifft oder Gleichgewichtsüberlegungen verschiedenster Art zu Grunde legt. Das ist analog zur Frage des Wettbewerbs für die Kanalisierung individueller Entscheidungen und hat damit zu tun, dass Makrophänomene nicht immer mit dem Verhalten typischer Akteure auf der Mikroebene korrespondieren. Repräsentative Agenten sind letztlich über Aggregationsvorüberlegungen abgeleitete Annahmen, die das Aggregat in theoretischen Zugängen auf möglichst einfache und konsistente Weise in seiner Regelhaftigleit widerspiegeln. Dahinter stehen sehr allgemeine Überlegungen über Aggregationsprobleme und -verfahren (Schlicht 1977, 1985), die häufig damit zu tun haben, dass es systematische qualitative Unterschiede von Mikro- und Makrorelationen gibt.

An industry might grow although all forms shrink in size, and the consumption habits of individual households might be influenced by other households, but no reference group effects are possible for the representative household. Examples such as these give only the simplest cases where the behavior of the typical agent deviates from that of the corresponding representative agent, but they illustrate already that micro and macro behavior might differ. (Schlicht 1985, S. $11 \mathrm{f}$.).

Die Formalisierung von ABM durch den analytischen Startpunkt auf der Ebene von Individuen, ihrem Verhalten und ihren komplexen Interaktionen stellt sicherlich eine Möglichkeit dar, um zu den Aggregaten zu gelangen, makroökonomische Phänomene auch in ihrer Eigenständigkeit zu rekonstruieren und Schlüsse daraus zu ziehen. Es stellt sich dennoch die Frage, in welchen Fällen die zweifellos sehr anspruchsvolle und für Ökonom_innen durchaus aufwendig zu erarbeitende und anzuwendende Methode der ABM notwendig und sinnvoll ist und unter welchen Umständen auch einfachere Methoden über Aggregationsüberlegungen rechtfertigungsfähig sind, um mit Phänomenen auf der Makro- oder allgemeiner irgendeiner Aggregatebene geeignet umzugehen. Vielfach kann es ausreichen, auf der Grundlage 
der isolierenden Methode, die auf Marshall und Keynes zurück geht, eigenständige Annahmen über Makrophänomene zu entwickeln und dabei Glättungs-, Eliminations- und Systemeffekte im Mikro-Makrozusammenhang zu berücksichtigen.

Diese Effekte können genau den qualitativen Unterschied von Mikro- und Makrorelationen ausmachen. Schlicht (1977, Kap. 5) gibt eine Reihe von Beispielen für derartige Phänomene. Glättungseffekte können auftreten, wenn einzelne typische Individuen nichtstetiges Nachfrageverhalten aufweisen, in der Aggregation sich aber eine geglättete, stetige Nachfragefunktion ergibt. Oder jede einzelne typische Unternehmung mag Bereiche sinkender Durchschnittskosten aufweisen (Nichtkonvexität der Produktionsmenge), was im Aggregat bei hinreichend vielen Unternehmungen aber „konvexifiziert“ wird, d.h. genauer, dass der nichtkonvexe Bereich verschwindet, wenn der Bereich der individuellen nichtkonvexen Produktionsmengen im Vergleich zur Größe des Marktes hinreichend klein ist. So ähnlich verhält es sich mit nichtkonvexen Präferenzen typischer Haushalte. Eliminationseffekte treten z. B. dann auf, wenn etwa die individuellen Nachfragen von den Preisen abhängen, die aggregierte Nachfrage aber nur noch vom Einkommen. Das ist eine entscheidende Überlegung zur Rechtfertigung und Interpretation der Keynesschen Konsumfunktion. Systemeffekte schließlich treten dann auf, wenn es kausale Interdependenzen auf der Makroebene gibt, was ja auch gerade ein zentraler Ausgangspunkt der ABM ist. Schlicht (1977, S. 92) gibt dazu das Beispiel, wie sich durch wechselseitige Abhängigkeiten Mikro-Sparfunktionen von der langfristigen Makro-Sparfunktion unterscheiden können.

Schlicht $(1977,1985)$ entfaltet das Argument im Detail, und es bleibt allein aus Gründen der Einfachheit der Modellierung zu klären, ob man bei der Analyse konkreter makroökonomischer Fragen in Kenntnis der Aggregationsphänomene (z. T. auch als Gleichgewichtsphänomene zu verstehen) unmittelbar mit Makro-Annahmen starten sollte oder mit der Ebene der Individuen, ihren tatsächlichen Verhaltensdispositionen und all den Verwobenheiten ihrer Entscheidungen und Handlungen. Da ABM zunächst nur eine Modellmethode darstellt und keinem unmittelbaren ökonomischen oder Modellierungsparadigma folgt, muss bei der zweiten Variante natürlich ein Hauptaugenmerk darauf liegen, die tatsächlich relevanten Zusammenhänge individuellen Entscheidens, Interagierens einschließlich der Regelhaftigkeiten zu erfassen. Das ist keineswegs trivial oder gar beliebig, sondern konstituiert die entscheidende Aufgabe, sinnvolle und für den jeweiligen Analysezweck notwendige Zusammenhänge zu erfassen sowie daraus Verhaltensannahmen zu begründen.

Jeder Satz von Beobachtungen kann bekanntlich unendlich viele Kausalerklärungen bekommen, und wenn man mehr beabsichtigt als eine reine ex-post-Rationalisierung (und mehr als history friendly modeling), wird man tatsächliche Wirkungszusammenhänge identifizieren und begründen müssen. ABM bietet dazu einige Möglichkeiten durch bessere empirische Fundierung und Kalibrierung. Das aber ist nachrangig der zweite wichtige Schritt; der erste muss darin liegen, sinnvolle Annahmen über Verhalten und Systemdynamiken zu treffen. Das ist die entscheidende Vorarbeit vor Anwendung der zweifellos in relevanten Fällen dann eleganten, wenngleich komplexen Modellierungsmethode ABM. 


\section{Auswahl an Verhaltensannahmen}

Albert (1991, Kap. 1 und 2) weist darauf hin, dass ein deduktives (oder über Simulationen hergeleitetes, Anm. der Verf.) Argument nichts über den Wahrheitsgehalt seiner Komponenten aussagt, sondern nur logische Schlüsse aus dem Annahmensatz zulässt. Das ist soweit trivial und stimmt, ist allerdings auch zu allgemein in der Aussage als Kritik etwa an der Neoklassik, da es ja nicht nur um wahre Verhaltensgründe geht, sondern aus Isolationsgesichtspunkten u. U. auch die Optimierungshypothese durchaus eine sinnvolle Vereinfachung in speziellen Fällen darstellen kann. Das macht es aber doppelt anspruchsvoll, Verhaltensannahmen zu begründen. Sie müssen letztlich auf einem sozialpsychologischen Fundament aufbauen, erstens um konkrete Verhaltensbestimmungen der Menschen nicht nur aus einzelnen speziellen und nicht immer widerspruchsfreien Effekten (Status-quo-Orientierung, Risikowahrnehmung, Gleichheitspräferenz o. Ä.) abzuleiten, sondern dies mit einem übergreifenden Theorierahmen zu begründen. Und dieser Theorierahmen kann dann zweitens auch helfen zu beurteilen, unter welchen Umständen es im Zuge der Aggregation und Isolation auch zulässig sein kann, vereinfachte und zudem auch u. U. gänzlich unterschiedliche Annahmen auf verschiedenen Aggregatebenen zu verwenden.

Hinsichtlich der Frage nach einem übergreifenden Theorierahmen gibt es bereits einige Ansätze. Hier seien nur zwei Vorschläge beispielhaft genannt, die beide i. w. S. evolutionsökonomischen Überlegungen folgen. Der erste wird inzwischen mit dem Label Evolutionary Behavioral Economics bezeichnet (Burnham et al. 2016). Der zweite Ansatz kann als gestalttheoretisch inspirierter Ansatz umschrieben werden (Kubon-Gilke et al. 2018), wobei auch dieser Theorierahmen evolutionstheoretisch argumentiert, aber den Invarianzen der Evolution und naturgesetzlichen Gleichgewichtsphänomenen z. B. in der Gehirnorganisation und damit der Wahrnehmung der Menschen größere Bedeutung zumisst, während der erste Ansatz die Selektionsmechanismen in den Vordergrund stellt und spezielle Annahmen insbesondere zu Interaktionen und zum Lernen verwendet. Diese verschiedenen Theorierahmungen gilt es immanent zu diskutieren. Solange der Diskurs dazu und anderen Vorstellungen übergreifender Theorien unterbleibt, wird ABM zwar eine sehr sinnvolle und durchaus aufwendige Ergänzung des Modellinstrumentariums darstellen, aber es fehlt dennoch ein entscheidender Schritt bei der Frage, unter welchen Umständen genau sich diese Methode als sowohl einfache als auch inhaltlich am besten passende identifizieren lässt.

In ihrem 1965 in deutscher Sprache erschienenen Buch ,Doktrinen der Wirtschaftswissenschaft' gibt Joan Robinson ein vernichtendes Urteil über den damaligen Stand der Ökonomik, indem sie formuliert: „Am einen Bein ungeprüfte Hypothesen, am anderen unprüfbare Slogans - so humpelt die Nationalökonomie daher" (Robinson 1965, S. 35). Sie bemängelt zweierlei: erstens, dass es in der Ökonomik keine guten empirischen Widerlegungsmöglichkeiten gäbe und dass sich zweitens Theoretiker_innen nie von Denkgewohnheiten ihrer jeweiligen Zuordnung zu einer bestimmten Schule und auch nicht von ,eingesickerten“ ethischen Vorurteilen frei machen könnten. Im Hinblick auf empirische Überprüfbarkeit hat es zweifellos in der Zwischenzeit deutliche Weiterentwicklungen etwa über kontrollierte Experimente, neue ökonometrische Verfahren und auch speziell durch die ABM gegeben. Den- 
noch verbleibt das von Robinson beschriebene Problem, dass es in der Ökonomik nach wie vor stark auf die Interpretation von Ergebnissen ankommt, die wiederum von Codes, Sprach- und Denkgewohnheiten verschiedener ökonomischer Schulen geprägt sind. Ludwik Fleck (2011) bezeichnete dies als Denkstile, denen dann Denkkollektive folgten. Die stärkere Öffnung für heterodoxe Ansätze bzw. der Weg zu einer pluralen Ökonomik eröffnet Diskurse, um solche Denkstile aufzudecken und die expliziten wie impliziten Annahmen, Begrifflichkeiten und Interpretationen offenzulegen und in den Diskurs zu bringen. Es ist ein nicht zu unterschätzender zusätzlicher Vorteil der ABM, dass ein für viele Fragen offener, nicht einzelnen Paradigmen folgender methodischer Weg diesen Diskurs durch die klare Struktur der Vorgehensweise erleichtern kann.

\section{Literatur}

Albert, H. (1991). Traktat über kritische Vernunft (5. Aufl.). Tübingen: Mohr (Siebeck). 1. Auflage 1968 Alchian, A. A. (1950). Uncertainty, evolution, and economic theory. Journal of Political Economy, 58(3), 211-221. https://doi.org/10.1086/256940.

Burnham, T.C., Lea, S.E. G., Bell, A., Gintis, H., Glimcher, P.W., Kurzban, R., Lades, L., McCabe, K., Panchatanathan, K., Teschl, M., \& Witt, U. (2016). Evolutionary behavioral economics. In D. S. Wilson \& A. Kirman (Hrsg.), Complexity and evolution: Toward a new synthesis for economics. Strüngmann Forum Reports, (Bd. 19, S. 113-144). Cambridge: MIT Press.

Fleck, L. (2011). Denkstile und Tatsachen. Gesammelte Schriften und Zeugnisse. Frankfurt a.M.: Suhrkamp.

Kirman, A. (1992). Whom or what does the representative agent represent? Journal of Economic Perspectives, $6,117-136$.

Kubon-Gilke, G., et al. (2018). Gestalten der Sozialpolitik. Theoretische Grundlegungen und Anwendungsbeispiele. Marburg: Metropolis.

Robinson, J. (1965). Doktrinen der Wirtschaftswissenschaft. Eine Auseinandersetzung mit ihren Grundgedanken und Ideologien. München: C. H. Beck.

Schlicht, E. (1977). Grundlagen der ökonomischen Analyse. Reinbek: Rowohlt.

Schlicht, E. (1985). Isolation and aggregation in economics. Heidelberg, Berlin, New York: Springer. 